VOL. 37 (1988) [321-322]

\title{
JORDAN DERIVATIONS ON PRIME RINGS
}

\author{
M. BREŠAR AND J. VUKMAN
}

The purpose of this paper is to present a brief proof of the well known result of Herstein which states that any Jordan derivation on a prime ring with characteristic not two is a derivation.

Throughout this paper all rings will be associative. We shall denote by $Z(R)$ the centre of a ring $R$. An additive mapping $D: R \rightarrow R$ will be called a derivation if $D(x y)=D(x) y+x D(y)$ holds for all pairs $x, y \in R$. We call an additive mapping $D: R \rightarrow R$ a Jordan derivation if $D\left(x^{2}\right)=D(x) x+x D(x)$ holds for all $x \in R$. Obviously, every derivation is a Jordan derivation. The converse is in general not true. In this paper we present an alternative proof of the following theorem.

THEOREM 1. (Herstein [1]) Let $R$ be a prime ring with characteristic not two and let $D: R \rightarrow R$ be a Jordan derivation. Then $D$ is a derivation.

For the proof of Theorem 1 we need several steps. First we have

Proposition 2. Let $R$ be a ring with characteristic different from two and let $D: R \rightarrow R$ be a Jordan derivation. Then the following hold:

(1) $D(a b+b a)=D(a) b+a D(b)+D(b) a+b D(a)$ for all $a, b \in R$;

(2) $D(a b a)=D(a) b a+a D(b) a+a b D(a)$ for all $a, b \in R$;

(3) $\quad D(a b c+c b a)=D(a) b c+a D(b) c+a b D(c)+D(c) b a+c D(b) a+c b D(a)$ for all $a, b, c \in R$.

(1) is immediate. The proof of (2) is not difficult and can be found in [1] and [2]. (3) follows immediately from (2). For any Jordan derivation $D$ we shall write $a^{b}$ for $D(a b)-D(a) b-a D(b)$. From (1) in Proposition 2 we see that

$$
b^{a}=-a^{b}
$$

holds for all $a, b \in R$. It is easy to see that for all $a, b, c \in R$ the relation

$$
a^{b+c}=a^{b}+a^{c}
$$

holds. All is prepared for the proof of the result below.

Received 10 June 1987

This work was supported by the Research Council of Slovenia, Yugoslavia

Copyright Clearance Centre, Inc. Serial-fee code: 0004-9729/88 \$A2.00+0.00. 
Theorem 3. Let $R$ be a ring of characteristic not two, and let $D: R \rightarrow R$ be a Jordan derivation. In this case for all $a, b, r \in R$ we have

$$
a^{b} r(a b-b a)+(a b-b a) r a^{b}=0
$$

Proof of Theorem 3: Let us write $W$ for $a b r b a+b a r a b$. Then by (2) of Proposition 2 we obtain

$$
\begin{aligned}
D(W) & =D(a(b r b) a+b(a r a) b) \\
& =D(a) b r b a+a D(b r b) a+a b r b D(a)+D(b) a r a b+b D(a r a) b+b a r a D(b) \\
& =D(a) b r b a+a D(b) r b a+a b D(r) b a+a b r D(b) a+a b r b D(a) \\
& +D(b) a r a b+b D(a) r a b+b a D(r) a b+b a r D(a) b+b a r a D(b) .
\end{aligned}
$$

On the other hand we obtain using (3) of Proposition 2

$$
\begin{aligned}
D(W) & =D((a b) r(b a)+(b a) r(a b)) \\
& =D(a b) r b a+a b D(r) b a+a b r D(b a)+D(b a) r a b+b a D(r) a b+b a r D(a b) .
\end{aligned}
$$

By comparing and using (1) we obtain (3). The proof of the theorem is complete.

The proof of Theorem 1 is an almost immediate consequence of Theorem 3 and Lemma 3.10 in [2].

Proof of Theorem 1: Let $a$ and $b$ be fixed elements from $R$. If $a b \neq b a$ then from Theorem 3 and Lemma 3.10 in [2] one obtains immediately that $a^{b}=0$. If $a$ and $b$ are both in $Z(R)$ then $a^{b}=0$ follows from (1) in Proposition 2. It remains to prove that $a^{b}=0$ also in the case when $a \notin Z(R)$ and $b \in Z(R)$. There exists $c \in R$ such that $a c \neq c a$. Since $a c \neq c a$ and $a(b+c) \neq(b+c) a$ we have $a^{c}=0$ and $a^{b+c}=0$. Then we obtain using (2) $0=a^{b+c}=a^{b}+a^{c}=a^{b}$. The proof of the theorem is complete.

\section{REFERENCES}

[1] I.N. Herstein, 'Jordan derivations of prime rings', Proc. Amer. Math. Soc. 8 (1957), 1104-1110.

[2] I.N. Herstein, Topics in ring theory (Chicago lectures in mathematics, 1969).

Cankarjeva 24

62000 Maribor

Yugoslavia
University of Maribor

VEK $\dot{S}$

Razlagova 14

62000 Maribor

Yugoslavia 